\title{
DINÂMICA DA OCUPAÇÃO E DA QUALIFICAÇÃO DA MÃO DE OBRA NA AGROPECUÁRIA BRASILEIRA ${ }^{1}$
}

\author{
Katy Maia $^{2}$ \\ Rossana Lott Rodrigues ${ }^{3}$ \\ Solange de Cássia Inforzato de Souza \\ Antonio Carlos Moretto ${ }^{5}$ \\ Ricardo Kureski
}

\begin{abstract}
Resumo: Este artigo analisa os efeitos da mudança tecnológica, do consumo final e da abertura comercial sobre a estrutura de emprego do Brasil e do setor agropecuário, por nível de qualificação, entre 1985 e 2003. Para tanto, foram utilizados dados de matrizes de insumo-produto e das PNADs - Pesquisa Nacional por Amostra de Domicílios dos respectivos anos. Os resultados mostraram importantes mudanças na estrutura de emprego do setor agropecuário neste período, em favor dos trabalhadores qualificados, ainda que em menor proporção em relação aos outros setores da economia, provocada pela mudança tecnológica e pelo comércio exterior. O setor agropecuário revelou-se dinâmico em sua estrutura de emprego, beneficiando mais o trabalho qualificado com elevada produtividade.
\end{abstract}

Palavras-chave: liberalização comercial, setor agropecuário, qualificação da mão de obra, mudança tecnológica, produtividade do trabalho.

Abstract: The article analyses the effect of trade liberalization, of final consumption and of technological change on employment structure of Brazil and sector agro cattle raising, by skill level, between 1985 and 2003. Therefore, were used input-output and PNAD data of respective years. The results obtained show important change on the employment structure of sector agro cattle raising in this period, in favor the skilled workers, yet that in less proportion relative to others economic sectors, caused by technological change and trade foreign. The agro cattle raising sector revealed dynamic in its employment structure, benefiting more the skill labor with raised labor productivity.

Key words: trade liberalization; agro cattle raising sector; skilled workers; technological change; labor productivity

Recebido em: 22/03/11. Aceito em: 07/06/11.

Professora Doutora pela UnB-DF. Professora da Universidade Estadual de Londrina. E-mail: katymaia@uel.br. Professora Doutora pela ESALQ-SP. Professora da Universidade Estadual de Londrina. E-mail: rlott@uel.br. Professora Doutora pela PUC-SP. Professora da Universidade Estadual de Londrina. E-mail: soinfor@uel.br. Professor Doutor pela ESALQ-SP. Professor da Universidade Estadual de Londrina. E-mail: acmoretto@uel.br.

6 Professor Doutor pela UFPR. Professor da Pontifícia Universidade Católica do Paraná. Pesquisador do Instituto Paranaense de Desenvolvimento Econômico e Social. E-mail: kureski@ipardes.pr.gov.br. 


\section{Introdução}

A literatura econômica sobre o mercado de trabalho, nos últimos anos, vem mostrando grande interesse pela questão do emprego e da qualificação da mão de obra. A dinâmica desse mercado no âmbito tecnológico tem repercutido em todos os setores produtivos das economias, especialmente no agropecuário. Por outro lado, a maior integração entre as nações e a formação de blocos econômicos têm estreitado ainda mais o comércio internacional.

Existe um consenso nessa literatura de que tem havido, nos últimos anos, queda da demanda por mão de obra menos qualificada em consequência do comércio internacional e da mudança tecnológica ${ }^{7}$. No entanto, há controvérsia entre os pesquisadores quanto à intensidade dessas causas. Para muitos, a queda da demanda por mão de obra de baixa qualificação é causada, primordialmente, pela mudança tecnológica, havendo pouca influência da abertura comercial ${ }^{8}$. Outros pesquisadores destacam a relevância do comércio internacional frente à mudança tecnológica ${ }^{9}$. $\mathrm{O}$ debate sobre essa questão está longe de acabar, pois ainda há vários estudos se aprofundando no tema.

Algumas razões nos levam a investigar o caso do Brasil, especialmente o setor agropecuário, que tem tido importante papel no seu desenvolvimento econômico. Em que pesem as situações conjunturais adversas, a agropecuária nacional apresentou, nos últimos 14 anos, taxa de crescimento real anual de seu Produto Interno Bruto (PIB) superior ao desempenho global da economia brasileira, 2,4 pontos percentuais e $1,11 \%$ ao ano, respectivamente (AVELLAR e VILELA, 2006). Esse desempenho do setor, tanto interna (crescimento da renda) quanto externamente (superávits crescentes na balança comercial, diversificação na pauta de exportação e liderança mundial na comercialização de diversos produtos) foi fruto da ampliação, a partir da década de 1990,

\footnotetext{
Katz e Murphy (1992), Bermam, Bound e Griliches (1994), Machin (1996), Nickell e Bell (1995).

8 Greenhalgh, Gregory e Zissimos (1998), Berman, Bound e Machin (1998), e Desjonqueres, Machin e Van Reenen (1999)

9 Wood (1994), Sachs e Shatz (1994) e Haskel e Slaughter (1999).
} 
Katy Maia, Rossana Lott Rodrigues, Solange de Cássia Inforzato de Souza, Antonio Carlos Moretto \& Ricardo Kureski

dos investimentos em tecnologia, que intensificaram um processo que se iniciou ainda na década de 1960, tendo provocado dois efeitos já conhecidos: a) aumento da produtividade total dos fatores, inclusive da mão de obra e, consequentemente, da competitividade do setor; e b) queda no número de pessoas ocupadas na agropecuária.

O país passou, no início dos anos 90, por um rápido processo de liberalização comercial, o que tornou sua economia mais exposta à concorrência internacional e sujeita às inúmeras mudanças, inclusive no mercado de trabalho. Alguns analistas têm investigado a influência da abertura comercial no emprego, como, por exemplo, Moreira e Najberg (1997), Arbache e Corseuil (2000) e Machado e Moreira (2000) ${ }^{10}$. Vale lembrar ainda que grande parcela da mão de obra brasileira é composta por trabalhadores de baixa qualificação (muitos do setor agropecuário), supostamente os mais afetados pela mudança tecnológica e pela abertura comercial. Apesar disso, o Brasil tornou-se uma proeminente economia que vem se destacando entre as economias emergentes com projeção mundial, devido, principalmente, ao desempenho de suas commodities no comércio internacional e ao seu crescimento econômico nos últimos anos. Neste contexto, qual foi o efeito do comércio exterior, do consumo e da mudança tecnológica no emprego por qualificação de mão de obra no Brasil?

Diante disso, este trabalho objetivou medir o impacto do comércio internacional, da mudança tecnológica e do consumo final sobre a estrutura do emprego por nível de qualificação no Brasil, e em seu setor agropecuário, no período entre 1985 e 2003, com base nos dados das matrizes de insumo-produto e da Pesquisa Nacional por Amostra de Domicílios (PNAD), ambas do Instituto Brasileiro de Geografia e Estatística (IBGE).

O artigo está dividido em três seções, além da introdução. Na segunda seção, detalha-se a metodologia adaptada aos dados brasileiros. $\mathrm{Na}$

${ }_{10}$ As três obras citadas mostraram, em síntese, que o aumento dos fluxos comerciais causou mudanças expressivas na participação do emprego, embora tenham adotado metodologias distintas à do presente artigo. 
terceira, são analisados os resultados para a economia brasileira e para o setor agropecuário e, no final, estão as conclusões do estudo.

\section{Metodologia}

A metodologia utilizada no presente trabalho foi inspirada no estudo de Greenhalgh et al. (1998), que investigou a mudança na estrutura da demanda de mão de obra do Reino Unido.

\subsection{Fonte de dados}

Como base de dados para o caso brasileiro, foram usadas a matriz de insumo-produto nacional de 1985 (IBGE, 1987) e a Pesquisa Nacional por Amostra de Domicílios - PNAD (IBGE, 1987a e 2004a), além da matriz de insumo-produto de 2003, estimada por Kureski e Nuñez (2007). É importante lembrar que o IBGE não calculou as matrizes de insumoproduto no período de 1997 a 2004. Ademais, a matriz de 2005 e a dos anos seguintes, calculadas pelo IBGE, apresentam incompatibilidades com a de 1985. Por isso, neste estudo, os autores optaram estudar o período 1985-2003. Os valores correntes da matriz de insumo-produto de 1985 foram atualizados para os de 2003, tendo como base os índices de preços por produto (nível 80) elaborados pelo IBGE. Assim, foi possível calcular a variação da produção no período a preços constantes de 2003.

No cálculo da produção do setor agropecuário brasileiro e do país, mostrado a seguir, foi utilizada a classificação do IBGE, que engloba 42 setores da matriz de insumo-produto de 1985 (IBGE, 1987). Posteriormente, a fim de adaptarmos os resultados da produção aos dados de emprego, agregamos os 42 setores de atividade em 31. Esse procedimento foi necessário para evitar repetições dos códigos de três dígitos na compatibilização com os códigos de dois dígitos. 
Katy Maia, Rossana Lott Rodrigues, Solange de Cássia Inforzato de Souza, Antonio Carlos Moretto \& Ricardo Kureski

A partir dos dados de emprego por grau de escolaridade, que serviram como proxy para a qualificação da mão de obra, calculamos as proporções dos trabalhadores por grau de escolaridade com os microdados das PNAD ${ }^{11}$. Em seguida, aplicamos essas proporções no total de mão de obra das matrizes de insumo-produto. Assim, estabeleceu-se a mão de obra segundo sua qualificação.

O grau de escolaridade foi classificado em cinco níveis, conforme os anos completos de estudo do trabalhador ${ }^{12}$. Os quatro primeiros níveis consideram a mão de obra menos qualificada, abrangendo trabalhadores com até 11 anos de estudo; o último nível, referente aos trabalhadores com curso superior incompleto ou mais, representa a mão de obra qualificada.

\subsection{Modelo Matemático}

Para cada um dos anos estudados, 1985 e 2003, foi criada uma matriz, $\mathbf{N}$, que contém dados sobre emprego, de acordo com os cinco níveis de escolaridade e os 31 setores de atividade. Essas matrizes foram utilizadas com o objetivo de se considerar a mudança na estrutura de qualificação do emprego decorrente dos efeitos da mudança no consumo final, da liberalização comercial e da mudança tecnológica.

Da matriz $\mathbf{N}$, derivamos as matrizes de coeficientes técnicos do emprego direto, $\mathbf{n}$, para cada ano pesquisado, as quais representam o insumo de cada tipo de qualificação requerido para se produzir uma unidade de produto em cada setor de atividade. Agregamos a matriz $\mathbf{N}$ entre os setores, visando a produzir um vetor de emprego por grau de escolaridade N. Assim:

$N=\mathbf{n} X$

${ }_{11}$ Selecionamos indivíduos ocupados por atividade e por grau de escolaridade.

12 Sem escolaridade, 1 a 4 anos de estudo, 5 a 8 anos de estudo, 9 a 11 anos de estudo, e mais de 11 anos de estudo. 
em que $X$ é o vetor com o valor bruto da produção total por setor de atividade e $\mathbf{n}$ é a matriz de insumo de trabalho por tipo de qualificação, conforme o grau de escolaridade necessário à produção de uma unidade de produto por setor de atividade.

Na análise da matriz de insumo-produto, a identidade padrão do produto bruto corresponde a:

$X=\mathbf{A}^{d} X+\mathbf{S}\left(C^{d}+F^{d}+E\right)$

$\mathbf{A}^{d}$ é a matriz de coeficientes técnicos intersetoriais domésticos, ou seja, o produto da matriz de Market Share e a matriz de coeficientes técnicos de insumos nacionais. S, considerada constante, é a matriz de Market Share. $C^{d}$ é o vetor do fluxo de bens domésticos para o consumo final por setor, isto é, a soma dos vetores do consumo doméstico das famílias e do consumo doméstico da administração pública. $F^{d}$ é o vetor do fluxo de bens de capital por setor, ou seja, a soma dos vetores da formação bruta de capital fixo doméstico e da variação de estoque doméstico. Finalmente, $E$ corresponde ao vetor de exportação por setor. Consideramos a penetração das importações na produção de bens intermediários e finais. Dessa forma, o produto bruto passa a ser expresso por:

$$
X=(\mathbf{h} * \mathbf{A}) X+c * \mathbf{S} C+f * \mathbf{S} F+\mathbf{S} E
$$

A é a matriz dos coeficientes técnicos intersetoriais totais, ou seja, o produto da matriz de Market Share e a matriz de coeficientes técnicos de insumos totais; $\mathbf{h}$ é a matriz das proporções da demanda doméstica por bens intermediários sobre a demanda total por bens intermediários. Assim, $\mathbf{h} * \mathbf{A}$ é o produto de elemento por elemento das matrizes $\mathbf{h}$ e $\mathbf{A}$, ou seja, é a demanda de bens intermediários nacionais. O vetor $c$ representa as proporções do consumo final doméstico sobre o consumo final total e $\mathbf{S} C$ é o vetor do consumo final. $\operatorname{Logo}, c * \mathbf{S} C$ é o produto de elemento por elemento dos vetores $c$ e $\mathbf{S} C$. Da mesma forma, $f * \mathbf{S} F$ é o produto de elemento por elemento do vetor $f$ das proporções da demanda 
Katy Maia, Rossana Lott Rodrigues, Solange de Cássia Inforzato de Souza, Antonio Carlos Moretto \& Ricardo Kureski

doméstica de bens de capital sobre a demanda de bens de capital total e do vetor $\mathbf{S} F$ da demanda de bens de capital. Finalmente, o último termo $\mathbf{S} E$ corresponde ao vetor da exportação.

A solução da matriz de insumo-produto básica para o produto por setor é:

$X=(I-\mathbf{h} * \mathbf{A})^{-1}\left(c * \mathbf{S} C+f^{*} \mathbf{S} F+\mathbf{S} E\right)$

Ao substituir a equação (4) na equação (1), pode-se determinar o emprego total conforme a qualificação da mão de obra, ou seja, o seu grau de escolaridade e conforme o produto segundo sua composição, demanda final e intermediária:

$N=\mathbf{n} X=\mathbf{n}(I-\mathbf{h} * \mathbf{A})^{-1}(c * \mathbf{S} C+f * \mathbf{S} F+\mathbf{S} E)$

Como as expressões (1) e (5) ocorrem em qualquer período, podem ser diferenciadas com vistas a fornecer as mudanças ao longo do tempo. Tomando a diferença da equação (1), tem-se:

$\Delta N=\mathbf{n}_{t} X_{t}-\mathbf{n}_{0} X_{0}$

em que o índice 0 representa o período inicial e $t$ o período final. Essa mudança no emprego por grau de escolaridade pode ser vista em termos de mudança no produto e de mudança nos requerimentos de emprego por unidade do produto, o que resulta em:

$\Delta N=\mathbf{n} \Delta X+\Delta \mathbf{n} X$

em que $\mathbf{n}=\left(\mathbf{n}_{0}+\mathbf{n}_{t}\right) / 2$ e $X=\left(X_{0}+X_{t}\right) / 2$

Para decompormos a mudança no produto conforme suas origens, tomamos a diferença da expressão (4):

$$
\begin{aligned}
& \Delta X=X_{t}-X_{0} \\
& =\left(I-\mathbf{h}_{t}^{*} \mathbf{A}\right)^{-1}\left(\mathbf{c}_{t}^{*} \mathbf{S}_{t} C_{t}+f_{t}^{*} \mathbf{S}_{t} F_{t}+\mathbf{S}_{t} E_{t}\right)-\left(I-\mathbf{h}_{0}^{*} \mathbf{A}_{0}\right)^{-1}\left(c_{0} * \mathbf{S}_{0} C_{0}+f_{0}\right. \\
& \left.* \mathbf{S}_{0} F_{0}+\mathbf{S}_{0} E_{0}\right) \\
& =\mathbf{R}(c * \Delta \mathbf{S} C) \\
& \quad+\mathbf{R}(\Delta \mathbf{S} E)+\mathbf{R}\left(\Delta c^{*} \mathbf{S} C\right)+\mathbf{R}(\Delta \mathbf{h} * \mathbf{A}) X+\mathbf{R}(\Delta f * \mathbf{S} F) \\
& \quad+\mathbf{R}(\mathbf{h} * \Delta \mathbf{A}) X+\mathbf{R}(f * \Delta \mathbf{S} F)
\end{aligned}
$$


em que $\mathbf{R}=\left(I-\mathbf{h}^{*} \mathbf{A}\right)^{-1}=\left[\left(I-\mathbf{h}_{t} * \mathbf{A}_{t}\right)^{-1}+\left(I-\mathbf{h}_{0} * \mathbf{A}_{0}\right)^{-1}\right] / 2$

Substituindo a equação (8) na equação(7), referente à mudança no emprego, e reordenando os efeitos do consumo final, do comércio e da mudança tecnológica, obtém-se:

$$
\begin{aligned}
& \Delta N=\mathbf{n}\left[\mathbf{R}\left(c^{*} \Delta \mathbf{S} C\right)\right] \\
& \left.+\mathbf{n}\left[\mathbf{R}(\Delta \mathbf{S} E)+\mathbf{R}\left(\Delta c^{*} \mathbf{S} C\right)+\mathbf{R}\left(\Delta \mathbf{h}^{*} \mathbf{A}\right) X+\mathbf{R} \Delta f^{*} \mathbf{S} F\right)\right] \\
& +\mathbf{n}\left[\mathbf{R}\left(\mathbf{h}^{*} \Delta \mathbf{A}\right) X+\mathbf{R}\left(f^{*} \Delta \mathbf{S} F\right)\right]+\Delta \mathbf{n} X
\end{aligned}
$$

O primeiro termo do lado direito da equação acima fornece as mudanças no emprego atribuídas ao crescimento do consumo final. Na segunda linha, são indicados os efeitos do comércio sobre o emprego, com base no crescimento das exportações e na penetração das importações no consumo final, na demanda de bens intermediários e na de bens de capital. A terceira linha mostra os efeitos da mudança tecnológica.

\section{Resultados e Discussão}

\subsection{Análise da mudança na estrutura de emprego do Brasil}

A mudança total no emprego, por nível de qualificação e por origem, em números de trabalhadores empregados, no período examinado, pode ser observada na Tabela 2. O painel superior está dividido conforme as três origens da mudança no emprego, ou seja, o crescimento do consumo final, a exportação líquida e a mudança tecnológica. Os números da segunda coluna desse painel expressam a mudança total no emprego do país. No painel central, apresenta-se a mudança na mão de obra decorrente da exportação líquida e no painel inferior, o efeito decorrente da mudança tecnológica. 
Katy Maia, Rossana Lott Rodrigues, Solange de Cássia Inforzato de Souza, Antonio Carlos Moretto \& Ricardo Kureski

Tabela 2 - Mudança no emprego, por nível de qualificação e origem da mudança, em número de trabalhadores empregados, Brasil, $1985-2003$.

\begin{tabular}{|c|c|c|c|c|c|c|}
\hline \multirow[b]{2}{*}{$\begin{array}{c}\text { Nível de } \\
\text { Qualificação }\end{array}$} & \multirow[b]{2}{*}{$\begin{array}{c}\text { Mudança total no } \\
\text { emprego } \Delta N\end{array}$} & \multicolumn{5}{|c|}{ Origens da mudança no emprego } \\
\hline & & \multicolumn{2}{|c|}{$\begin{array}{c}\text { Consumo } \\
\mathbf{n} \mathbf{R}\left(c^{*} \Delta \mathbf{S} C\right)\end{array}$} & \multicolumn{2}{|c|}{$\begin{array}{c}\text { Exportação } \\
\text { líquida }\end{array}$} & $\begin{array}{c}\text { Mudança } \\
\text { tecnológica }\end{array}$ \\
\hline Menos Qualificados & 5.992 .610 & 41.307 .3 & & & 327.517 & -41.642 .288 \\
\hline Qualificados & 7.563 .812 & 5.611 .6 & & & 398.658 & 1.553 .477 \\
\hline Mudança absoluta & 13.556 .422 & 46.919 .0 & & & 726.175 & -40.088 .811 \\
\hline \multirow{3}{*}{$\begin{array}{l}\text { Nível de } \\
\text { Qualificação }\end{array}$} & \multirow{3}{*}{$\begin{array}{l}\text { Exportação } \\
\text { líquida }\end{array}$} & \multicolumn{5}{|c|}{ Origens da mudança no emprego devida ao comércio } \\
\hline & & Export. total & \multicolumn{2}{|c|}{$\begin{array}{l}\text { Import. } \\
\text { para }\end{array}$} & $\begin{array}{l}\text { Import. de bens } \\
\text { intermediários }\end{array}$ & $\begin{array}{l}\text { Import. de bens } \\
\text { de capital }\end{array}$ \\
\hline & & \multicolumn{3}{|c|}{$(\Delta c * \mathbf{S} C)$} & $\mathbf{n} \mathbf{R}(\Delta \mathbf{h} * \mathbf{A}) X$ & $\mathbf{n} \mathbf{R}(\Delta f * \mathbf{S} F)$ \\
\hline Menos Qualificados & 6.327 .517 & 10.849 .733 & -2.8 & 6.616 & -1.400 .078 & -305.521 \\
\hline Qualificados & 398.658 & 910.062 & -2 & 3.214 & -247.886 & -30.305 \\
\hline \multirow[t]{2}{*}{ Mudança absoluta } & 6.726 .175 & 11.759 .795 & -3.0 & 9.829 & -1.647 .964 & -335.826 \\
\hline & & \multicolumn{5}{|c|}{$\begin{array}{l}\text { Origens da mudança no emprego devida à mudança } \\
\text { tecnológica }\end{array}$} \\
\hline \multirow[t]{2}{*}{$\begin{array}{l}\text { Nível de } \\
\text { Qualificação }\end{array}$} & $\begin{array}{l}\text { Mudança } \\
\text { tecnológica }\end{array}$ & \multirow{2}{*}{\multicolumn{2}{|c|}{$\begin{array}{c}\text { Bens } \\
\text { intermediários } \\
\mathbf{n} \mathbf{R}(\mathbf{h} * \Delta \mathbf{A}) X\end{array}$}} & \multicolumn{2}{|c|}{ Bens de capital } & $\begin{array}{l}\text { Produtividade do } \\
\text { trabalho direto }\end{array}$ \\
\hline & & & & \multicolumn{2}{|c|}{$\mathbf{n} \mathbf{R}(f * \Delta F)$} & $\Delta \mathbf{n} X$ \\
\hline Menos Qualificados & -41.642 .288 & \multicolumn{2}{|c|}{3.680 .421} & \multicolumn{2}{|c|}{7.790 .747} & -53.113 .456 \\
\hline Qualificados & 1.553 .477 & \multicolumn{2}{|c|}{342.569} & \multicolumn{2}{|c|}{419.658} & 791.250 \\
\hline Mudança absoluta & -40.088 .811 & \multicolumn{2}{|c|}{4.022 .990} & \multicolumn{2}{|c|}{8.210 .405} & -52.322 .206 \\
\hline
\end{tabular}

Fonte: Elaboração própria. 
Inicialmente, examinando-se o painel superior, verifica-se que cerca de 13,56 milhões de postos de trabalho foram gerados pelo efeito positivo do crescimento do consumo final e do comércio exterior, em contrapartida aos efeitos negativos da mudança tecnológica. O consumo final gerou 46,92 milhões de postos de trabalho, e o comércio internacional, cerca de 6,73 milhões, que, em conjunto, perfazem 53,6 milhões de emprego criados nesse período.

O aumento no emprego em função do crescimento do consumo final doméstico criou cerca de 41,31 milhões de postos de trabalho menos qualificados e 5,61 milhões de postos qualificados. Tal desempenho confirma a relevância da variável consumo na geração de emprego do país e pode ser interpretado como reflexo da capacidade de a economia absorver as alterações no âmbito tecnológico e do comércio exterior, no período examinado. Resultados similares foram observados nas pesquisas de Greenhalgh et al. (1998), para o Reino Unido, e de Moreira e Najberg (1997), para o Brasil.

Os efeitos das exportações líquidas mantiveram-se positivos no período, em ambos os níveis de qualificação da mão de obra. Vale ressaltar que o efeito do comércio exterior no emprego pode ser tanto positivo quanto negativo, dependendo do nível de penetração das importações e das exportações da economia. Em períodos próximos à situação de abertura ou de elevado fluxo comercial, ou seja, no curto prazo, a tendência é o comércio reduzir o nível de emprego. Esta situação tende a se reverter na medida em que a economia se torna mais competitiva no mercado internacional, como ocorreu com a economia brasileira, segundo os resultados para o período 1985 e 2003 apresentados neste estudo.

Sob a perspectiva da qualificação da mão de obra, cabe enfatizar que uma parcela significativa $(88,8 \%)$ dos empregos gerados foi de ocupações menos qualificadas, apesar do incremento do emprego causado tanto pelo consumo final quanto pelas exportações líquidas.

No que tange ao efeito da mudança tecnológica, é possível observar a eliminação de 40,09 milhões de postos de trabalho, em contraposição aos 
Katy Maia, Rossana Lott Rodrigues, Solange de Cássia Inforzato de Souza, Antonio Carlos Moretto \& Ricardo Kureski

53,6 milhões criados pelos dois outros fatores de origem de mudança. Esses resultados corroboram a tendência de que as novas tecnologias reduzem de forma intensa os requisitos de mão de obra por unidade de produto, e mais, eliminam postos de trabalho de baixa qualificação $(41,64$ milhões). Tal desempenho é explicado pelo fato de as novas tecnologias caracterizarem-se como poupadoras de mão de obra menos qualificada e absorvedoras de mão de obra qualificada. Essa característica tende a se intensificar em ambiente pós-abertura comercial.

No painel central da Tabela 2, referente à decomposição do impacto do comércio exterior sobre o emprego, observa-se que o efeito da exportação total foi positivo em ambos os níveis de qualificação. Já o impacto das importações para consumo final, bens intermediários e bens de capital sobre o emprego foram todos negativos, como era esperado, tendo atingido maior número de postos de trabalho de baixa qualificação. O que chama a atenção é que as importações para consumo eliminaram mais postos de trabalho, relativamente às importações de bens intermediários e de capital, revelando certa dificuldade na transferência de tecnologia entre os países, apesar do processo de liberalização comercial.

O painel inferior da Tabela 2 mostra a decomposição do impacto da mudança tecnológica sobre o emprego no país. O impacto negativo da mudança tecnológica vem confirmar a tendência de redução dos requisitos de mão de obra por unidade de produto. Em outras palavras, a mudança tecnológica mostrou-se poupadora de mão de obra, o que implica, nesse caso, eliminação de postos de trabalho menos qualificados. Contudo, podese observar que, tanto as compras de bens intermediários, em ambos os níveis de qualificação, quanto as compras de bens de capital, beneficiaram o emprego, tendo havido criação de postos de trabalho em ambos os níveis de qualificação, principalmente no que se refere aos menos qualificados, visto que esses abrangem o maior contingente de mão de obra no Brasil. Isso indica que os bens intermediários e de capital adquiridos no período examinado geraram emprego no Brasil, dada a complementaridade entre tecnologia e mão de obra. 
Já o impacto da produtividade do trabalho direto sobre o emprego reduziu substancialmente o número de postos de trabalho menos qualificados, concomitantemente à geração de postos qualificados, embora em número bem menor. Em relação a esse aspecto, vale destacar outros fatores que podem ter contribuído para a incorporação de novas tecnologias, como a entrada de investimentos estrangeiros e os efeitos externos positivos advindos da abertura (Sarquis e Arbache, 2001). Convém ressaltar, ainda, que Moreira e Najberg (1997) encontraram em sua pesquisa um impacto positivo da produtividade sobre o emprego, o que sugere ter havido baixa eficiência no período logo após a abertura. Os resultados do presente estudo, ao contrário, mostram de forma clara que o impacto da produtividade do trabalho sobre o emprego foi negativo, indicando ganhos de eficiência nesse período. Destaca-se, contudo, que tais ganhos ocorreram no trabalho menos qualificado.

Uma forma mais interessante de verificar a mudança no emprego decorrente do impacto dos três referidos fatores é mostrada na Tabela 3 , em estrutura similar à da tabela anterior.

No painel superior, verifica-se que a mudança no emprego foi de $25,21 \%$, em virtude do efeito positivo do consumo final e do comércio exterior vis-à-vis os efeitos negativos da mudança tecnológica. O consumo final beneficiou o emprego em $87,25 \%$ e o comércio exterior em $12,51 \%$, enquanto a mudança tecnológica o reduziu em $74,55 \%$.

No que tange ao nível de qualificação da mão de obra nos postos de trabalho, o impacto positivo do crescimento do consumo final doméstico sobre o emprego foi muito maior entre os postos de trabalho qualificados $(148,62 \%)$, ao passo que o efeito positivo do comércio exterior foi pouco mais intenso em postos de trabalho de baixa qualificação (12,65\%). Já o efeito negativo da mudança tecnológica se refletiu na elevada redução de $83,28 \%$ do emprego menos qualificado. Por outro lado, o emprego qualificado foi favorecido em $41,14 \%$. Esses resultados mostram, de forma bastante clara, que os trabalhadores qualificados foram muito mais beneficiados nesse período. 
Katy Maia, Rossana Lott Rodrigues, Solange de Cássia Inforzato de Souza,

Antonio Carlos Moretto \& Ricardo Kureski

Ao examinar o painel central da Tabela 3 , referente à decomposição do impacto do comércio exterior no emprego, observa-se que o efeito positivo da exportação total foi mais intenso nos postos qualificados. Esse resultado é o oposto do esperado pela teoria de Heckscher-Ohlin, se considerarmos a dotação relativa do país. Tal comportamento, no entanto, pode ser explicado pela hipótese skill-enhancing-trade de Robbins (1994, 1996), observada em recentes pesquisas para países em desenvolvimento, mais precisamente pela contribuição da tecnologia via bens de capital e bens intermediários. No que tange ao impacto negativo das importações para o consumo final e de bens de capital sobre o emprego, verifica-se que ambos os níveis de qualificação foram afetados praticamente na mesma intensidade, já as importações de bens intermediários apresentaram uma redução maior do emprego qualificado $(-6,56 \%)$.

O painel inferior da Tabela 3 mostra a decomposição do impacto da mudança tecnológica sobre o emprego. A intensidade do efeito positivo das compras de bens intermediários foi maior em relação ao emprego qualificado $(9,07 \%)$. Já a intensidade do efeito positivo das compras de bens de capital foi maior no emprego menos qualificado (15,58\%). Este comportamento pode ser explicado pela complementaridade existente entre tecnologia e capital humano, como observado por Menezes Filho e Rodrigues Jr. (2001). 
Tabela 3 - Mudança no emprego, por nível de qualificação e origem da mudança, em percentual com base em 1985, Brasil, entre 1985 e 2003.

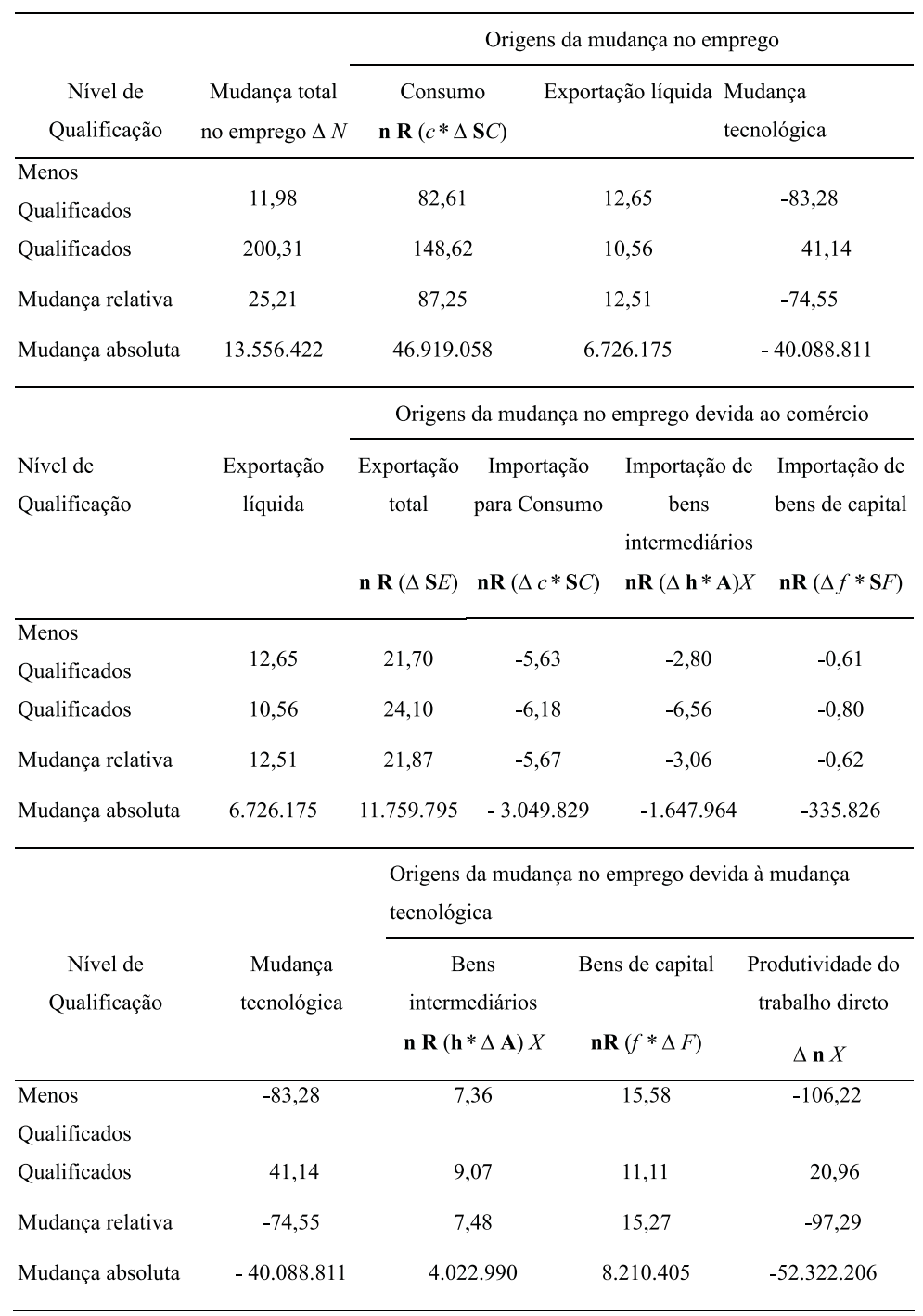

Fonte: Elaboração própria. 
Katy Maia, Rossana Lott Rodrigues, Solange de Cássia Inforzato de Souza, Antonio Carlos Moretto \& Ricardo Kureski

Em relação ao efeito da produtividade do trabalho direto sobre o emprego, a redução de postos menos qualificados foi baste significativa, perfazendo 106,22\%; no entanto, a criação de novos postos qualificados alcançou quase $21 \%$ no período. Logo, a geração de emprego advinda da mudança tecnológica deve-se, principalmente, à produtividade do trabalho direto, o que vem corroborar a hipótese dos efeitos externos positivos da abertura sobre o capital humano, testada por Sarquis e Arbache (2001).

O impacto negativo da mudança tecnológica indica que ocorreu significativo crescimento da produtividade após o processo de liberalização comercial. É importante ressaltar que o crescimento da produtividade da mão de obra está estreitamente ligado à reestruturação produtiva dos setores, a qual normalmente acompanha o processo recente de abertura comercial. Vale destacar ainda que diante da ameaça de maior concorrência, no início do processo de liberalização, muitas firmas brasileiras foram compelidas a buscar novas formas de produção.

Finalmente, salientamos que, ao considerarmos essas variações percentuais ocorridas no emprego do país, que indicam, claramente, benefícios maiores em favor do trabalho qualificado, devemos ter em mente que a proporção desse tipo de mão de obra no Brasil ainda é relativamente baixa, representando, no período analisado, em torno de $17 \%$ do seu total. A análise da mudança na estrutura do emprego no Brasil, no período de 1985 a 2003, aqui apresentada, servirá como parâmetro para examinarmos os resultados obtidos para o setor agropecuário do país, foco de análise deste estudo.

\subsection{Análise da mudança no emprego do setor agropecuário brasileiro}

A partir da multiplicação da matriz de coeficientes médios do emprego e da matriz diagonal da mudança na produção, foi possível calcularmos a mudança no emprego por setor e por nível de qualificação, segundo as origens de mudança: variação no consumo final, liberalização comercial 
e mudança tecnológica. Os resultados para o setor agropecuário estão nas Tabelas 4 e 5, em número de trabalhadores e em percentual, respectivamente.

O setor agropecuário, no período examinado, eliminou cerca de 4,41 milhões de posto de trabalho e, destes, apenas 613 eram qualificados. $\mathrm{O}$ Brasil há anos vem incorporando novas tecnologias à produção agropecuária, o que elevou extraordinariamente sua produtividade, incentivada pela abertura comercial, eliminando, assim, muitas de suas vagas de trabalho. 
Katy Maia, Rossana Lott Rodrigues, Solange de Cássia Inforzato de Souza, Antonio Carlos Moretto \& Ricardo Kureski

Tabela 4 - Mudança no emprego do setor agropecuário, por nível de qualificação e origem da mudança, em número de trabalhadores empregados, Brasil, 1985 - 2003.

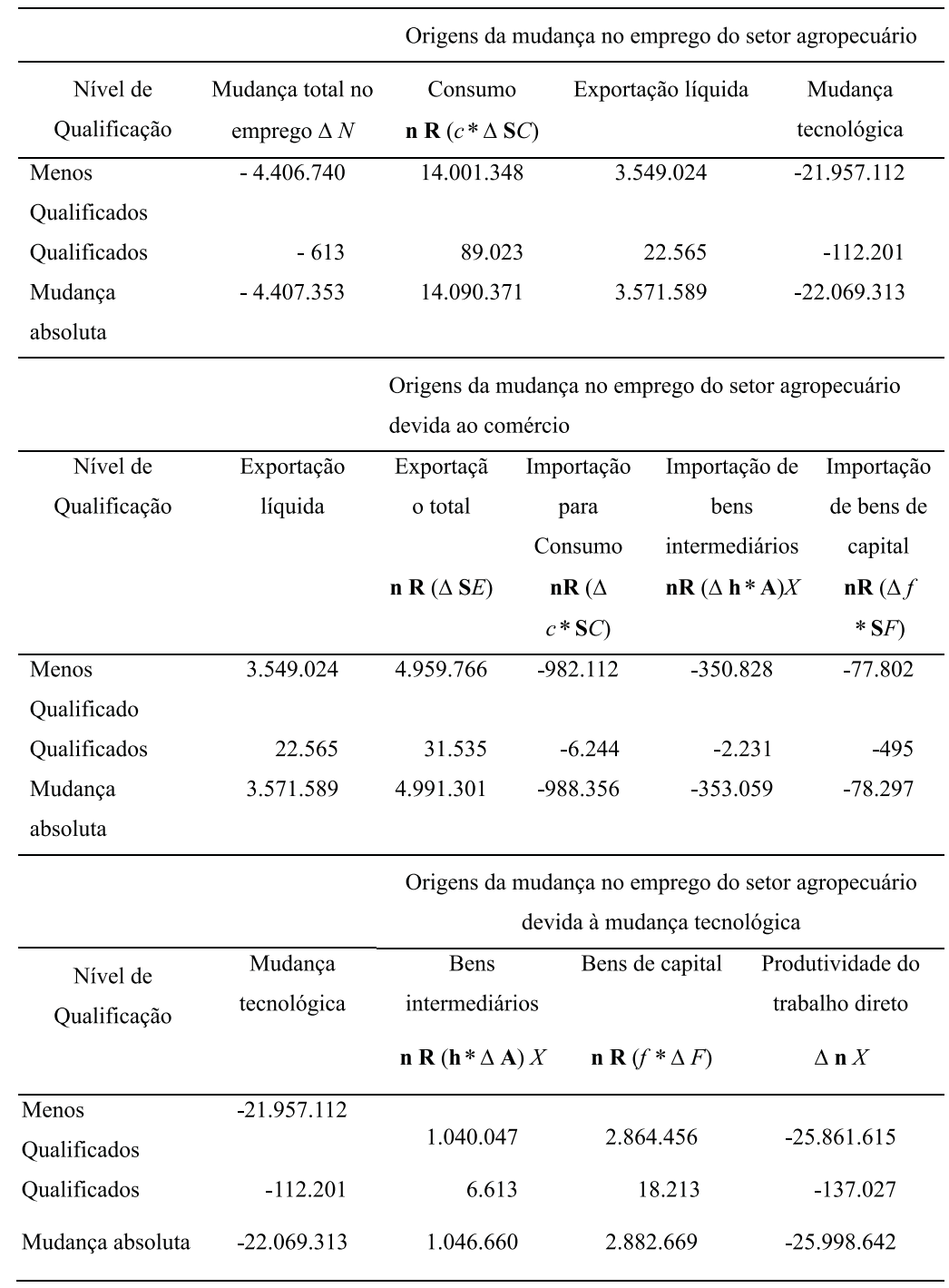

Fonte: Elaboração própria. 
É importante analisar os efeitos segundo a origem da mudança no emprego do setor agropecuário, isoladamente. Se observarmos apenas o impacto do consumo, pois entre as variáveis analisadas esta é a que mais gera emprego, verifica-se que ela chegou a gerar quase 14,10 milhões de postos de trabalho, sendo 89,02 mil qualificados. Entre 1985 e 2003, o emprego agropecuário representou $30,03 \%$ do total de empregos gerados por conta do consumo no país, entretanto, $33,89 \%$ do total de empregos gerados foram menos qualificados.

O comércio exterior gerou no referido período mais de 3,57 milhões de postos de trabalho, dos quais 22,56 mil eram qualificados, significando, assim, 53,1\% das ocupações geradas pelo setor exportador no Brasil. A exportação total criou em torno de 4,99 milhões de postos de trabalho, quase a totalidade (4,96 milhões) foi de baixa qualificação, o que pode ser justificado pelo fato de o Brasil nesse período ainda se apresentar intensivo em mão de obra menos qualificada, especialmente o setor agropecuário. Isso vem sendo alterado paulatinamente ao longo dos anos, dada a maior escolaridade da mão de obra brasileira. Na importação, seja para consumo, de bens intermediários, seja de bens de capital, a maior parcela da destruição de postos de trabalho foi a dos menos qualificados. Tal desempenho reflete também o movimento de maior escolarização da mão de obra brasileira.

A mudança tecnológica, em contrapartida, eliminou cerca de 22,07 milhões de vagas do setor, sendo que 112,20 mil eram vagas qualificadas, em contraposição à criação de ocupações já descritas pelos outros dois componentes analisados.

Em relação às três variáveis que compõem a mudança tecnológica neste estudo, observa-se que as compras domésticas de bens intermediários e as de capital impactaram positivamente a geração de emprego do setor agropecuário, em maior número os postos menos qualificados: 1,04 milhão e 2,86 milhões, respectivamente. Por outro lado, a produtividade do trabalho direto eliminou 26 milhões de postos de trabalho, dos quais a maioria (25,86 milhões) foi também de baixa qualificação. O que chama a atenção é a dimensão extremamente elevada, visto que no país, no 
Katy Maia, Rossana Lott Rodrigues, Solange de Cássia Inforzato de Souza, Antonio Carlos Moretto \& Ricardo Kureski

período examinado, foram eliminados 52,49 milhões de postos de trabalho, como mencionado anteriormente. Portanto, destes, 49,5\% eram do setor agropecuário. Pode-se considerar então que o setor agropecuário brasileiro se mostrou altamente dinâmico em termos tecnológicos, com elevada produtividade do trabalho, sendo um dos setores mais produtivos do Brasil.

Na Tabela 5, estão os resultados das variações percentuais da mudança no emprego para o setor agropecuário. No que se refere à geração de emprego neste setor, podemos observar que o consumo teve um grande impacto no mercado de mão de obra, pois apresentou crescimento de $82,3 \%$ no período analisado. Não obstante, o impacto foi maior ainda no emprego qualificado $(87,8 \%$ ), ainda que em menor proporção que o Brasil como um todo. Tal resultado indica que, apesar da mecanização, das barreiras comerciais e das dificuldades conjunturais, o setor agrícola ainda absorve muita mão de obra. Em termos relativos, o crescimento do trabalho qualificado superou o de menor qualificação, o que se reflete no uso de novas técnicas e na maior eficiência produtiva do setor.

Em relação ao comércio exterior, pode-se mostrar que o setor agropecuário apresentou excelente desempenho no que tange à geração de postos de trabalho no período examinado, haja vista que a exportação total apresentou crescimento de $29,2 \%$.

É importante destacar que, em relação à qualificação da mão de obra, o maior crescimento do emprego impulsionado pela exportação total foi para trabalhadores qualificados $(31,1 \%)$. Já os efeitos negativos da importação de bens para consumo foram mais expressivos do que os da importação de bens intermediários $(-5,8 \%$ e -2,1\%) e os da importação de bens de capital $(-0,5)$, sendo que os dois primeiros eliminaram relativamente mais postos qualificados, $-6,2$ e $-2,2$ respectivamente. Esses resultados mostram que a liberalização comercial afetou mais a importação para consumo. Daí a necessidade de uma política de importação mais voltada para bens de capital e bens intermediários.

No que se refere à mudança tecnológica, especificamente à aquisição doméstica de bens intermediários, o setor agropecuário apresentou 
crescimento razoável em relação à geração de emprego $(6,1 \%)$, principalmente de postos qualificados $(6,5 \%)$. Mas foram as compras de bens de capital que apresentaram maior crescimento $(16,8 \%)$, sendo também um crescimento mais expressivo de postos qualificados $(18,0 \%)$. Esses resultados indicam que a mudança tecnológica proporcionou, no período analisado, novas oportunidades aos trabalhadores do setor agropecuário, com destaque para a mão de obra mais qualificada, cujo crescimento foi claramente mais intenso. 
Katy Maia, Rossana Lott Rodrigues, Solange de Cássia Inforzato de Souza, Antonio Carlos Moretto \& Ricardo Kureski

Tabela 5 - Mudança no emprego do setor agropecuário, por nível de qualificação e origem da mudança, em percentual com base em 1985, Brasil, entre 1985 e 2003

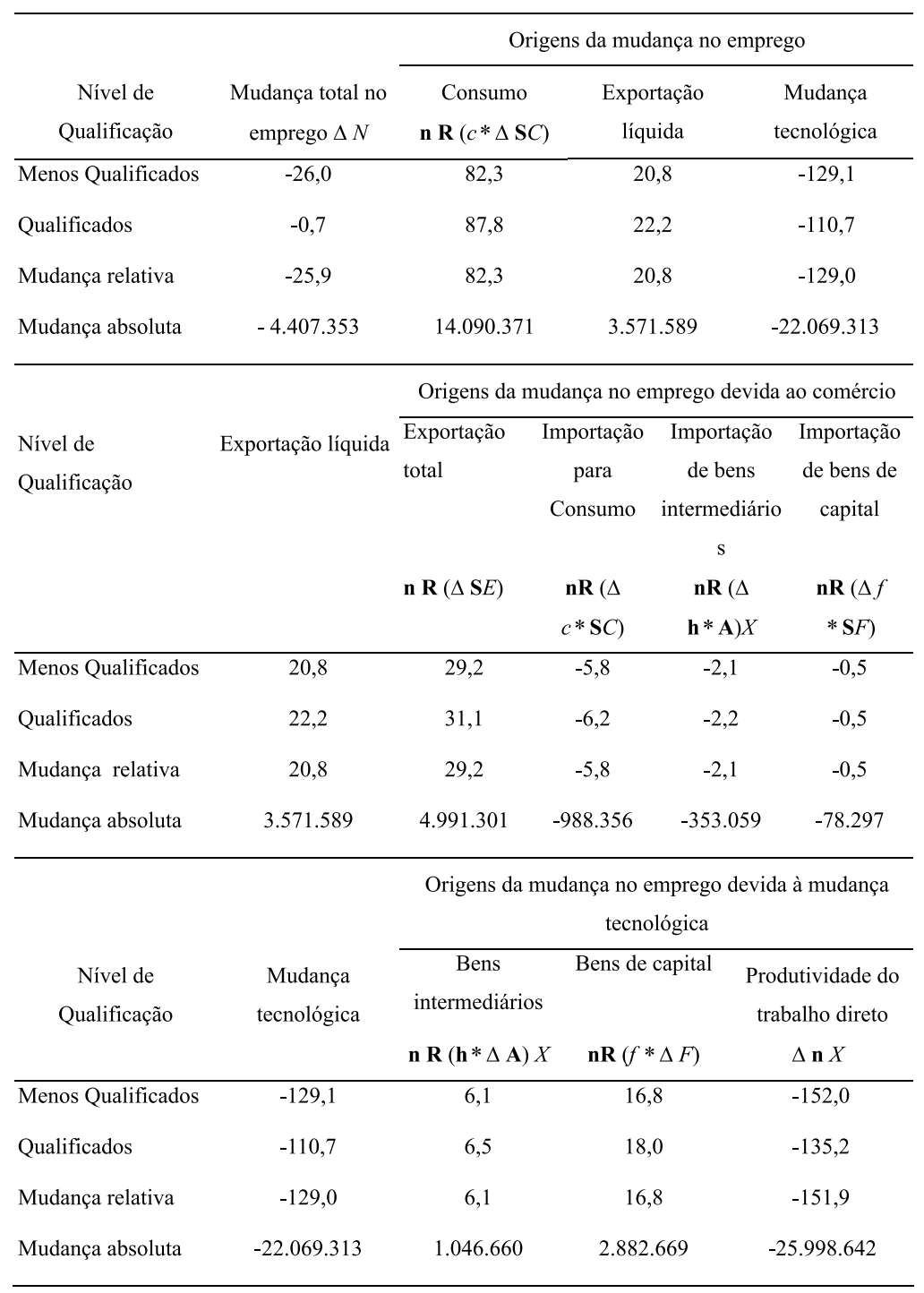

Fonte: Elaboração própria. 
Em relação à produtividade direta do trabalho, em termos relativos, houve redução de 151,9\% no emprego devido a esse fator. Quanto à qualificação das vagas eliminadas, $135,2 \%$ eram qualificadas e $152,0 \%$ menos qualificadas. Vale destacar que a agropecuária vem absorvendo, de forma bastante intensa, o avanço tecnológico e as novas técnicas de produção nos últimos anos, refletindo, assim, uma elevada produtividade do trabalho, que se confirma com os resultados obtidos.

O conjunto dos resultados obtidos mostra que, no período em questão, a agropecuária brasileira se tornou mais eficiente em seus processos produtivos e organizacionais, indicando maior produtividade da mão de obra, ao mesmo tempo em que o mercado de consumo e as exportações líquidas incrementaram relativamente os postos de trabalho agropecuários.

\section{Considerações finais}

A decomposição dos fatores que provocaram a mudança no emprego mostrou que o trabalhador qualificado foi beneficiado, vis-à-vis o menos qualificado, no período de 1985-2003, revelando que o processo de liberalização comercial, o consumo final e a mudança tecnológica contribuíram para estas transformações. Assim, vale detalhar as conclusões do estudo em relação ao quantum de emprego criado e eliminado e suas modificações relativas. Mas antes é preciso admitir quão difícil é a tarefa de isolar de forma analítica essas variáveis que afetam diretamente o capital humano. Neste contexto, a metodologia empregada procura se aproximar ao máximo do mundo real, sem perder de vista suas limitações, já que não contempla outras variáveis que podem afetar o mercado de trabalho.

Os resultados mostraram que, no agregado, o país cresceu em número de ocupações a despeito da eliminação ocorrida na agropecuária, indicando uma compensação de empregos conseguida pelos setores industrial e de serviço. Se em todos os setores o nível de qualificação cresceu vertiginosamente, a contribuição da agropecuária foi a menor 
Katy Maia, Rossana Lott Rodrigues, Solange de Cássia Inforzato de Souza, Antonio Carlos Moretto \& Ricardo Kureski

redução de destruição de ocupações qualificadas. Particularmente, o impacto da variação do consumo final sobre o emprego teve grande peso na geração de novos postos de trabalho, tanto nos setores em conjunto, como especificamente no agropecuário.

O efeito positivo do comércio exterior sobre o quantum de emprego foi verificado, embora relativamente o nível de emprego qualificado tenha crescido tanto quanto o de menos qualificado. O recorte no interior da variável "exportações líquidas" evidencia a importância das exportações em relação às importações para o incremento do emprego em todos os setores e na agropecuária. $\mathrm{O}$ setor exportador como gerador de emprego na agropecuária colabora mais do que proporcionalmente às importações e aos outros setores da economia.

Por outro lado, o resultado da mudança tecnológica no emprego foi negativo, atingindo profundamente os trabalhadores menos qualificados, o que não surpreende, visto que as inovações tecnológicas tendem a reduzir os requisitos de mão de obra por unidade de produto, eliminando postos de trabalho menos qualificados vis-à-vis à geração, em menor proporção, de postos qualificados.

O setor agropecuário, em especial, revelou-se bastante dinâmico em relação à sua estrutura de emprego por nível de qualificação, no período examinado. Considerando o impacto do consumo final, constatou-se crescimento maior de postos de trabalho qualificados, apesar do número bastante expressivo de postos menos qualificados gerados. O emprego da agropecuária também obteve um efeito positivo devido ao comércio exterior, embora em proporções bem menores ao do consumo final. Já o impacto da mudança tecnológica neste setor foi bastante profundo, eliminando milhões de postos de trabalho, devido à maior produtividade do trabalho direto, principalmente o menos qualificado. Contudo, as aquisições nacionais de bens intermediários e de capital compensaram parte dessa perda, gerando postos de trabalho mais qualificados.

Ao final, pode-se constatar que, entre 1985 e 2003, houve uma expressiva mudança na estrutura de emprego do país, em favor do trabalho 
qualificado, motivada pela liberalização comercial, consumo final e mudança tecnológica, enfatizando que o comércio exterior e as mudanças tecnológicas foram os grandes responsáveis pelas alterações na estrutura de emprego da agropecuária.

Cabe ainda destacar que os resultados desta pesquisa podem servir como um alerta para governantes e agentes de políticas públicas no sentido de mostrar as tendências da oferta e demanda de mão de obra por nível de qualificação no Brasil, especialmente no setor agropecuário, haja vista as referidas alterações na estrutura do emprego brasileiro.

\section{Referências}

ARBACHE, J. S.; CORSEUIL, C. H. L. Liberalização Comercial e Estrutura de Emprego e Salários. In: ENCONTRO NACIONAL DE ECONOMIA, 28., 2000, Campinas. Anais... Campinas: ANPEC, 2000.

AVELLAR, S. O. de C.; VILELA, P. S. Evolução do número de pessoas ocupadas na agropecuária brasileira no período de 1990 a 2004. Revista de Política Agrícola, ano XV, n. 2, p. 4-8, Abr./Maio/Jun. 2006.

BERMAN, E.; BOUND, J.; GRILICHES, Z. Changes in the Demand for Skilled Labor within U. S. Manufacturing: Evidence from the Annual Survey of Manufactures. Quarterly Journal of Economics, v. 109, p. 367-398, 1994.

BERMAN, E.; BOUND J.; MACHIN, S. Implications of Skill Biased Technological Change: International Evidence. Quarterly Journal of Economics, v.113, p.1245-1279, 1998.

DESJONQUERES, T.; MACHIN S.; VAN REENEN, J. Another Nail in the Coffin? Or Can the Trade Based Explanation of Changing Skill Strutures be Resurrected? Scandinavian Journal of Economics, v.101, p. 533-554, 1999. 
Katy Maia, Rossana Lott Rodrigues, Solange de Cássia Inforzato de Souza, Antonio Carlos Moretto \& Ricardo Kureski

GREENHALGH C.; GREGORY M.; ZISSIMOS, B. The Impact of Trade, Technological Change and Final Demand on the Skills Structure of UK Employment, Discussion Paper n. 29, Centre for Economic Performance, University of Oxford, 1998.

HASKEL, J.; SLAUGHTER, M. J. Trade, Technology and UK Wage Inequality. New York: NBER Working Paper n. 6978, 1999.

IBGE. Matriz de Insumo - Produto: Brasil - 1985. Rio de Janeiro: IBGE/ Departamento de Contas Nacionais, 1987.

. Pesquisa Nacional por Amostra de Domicílios: Brasil

- 1985. Rio de Janeiro: IBGE, 1987a.

Contas Nacionais: Brasil - 2004. Rio de Janeiro: IBGE/ Departamento de Contas Nacionais, 2004.

Pesquisa Nacional por Amostra de Domicílios: Brasil - 2003. Rio de Janeiro: IBGE, 2004a.

KATZ, F. L.; MURPHY, M. K. Changes in Relative Wages, 1963-1987: Supply and Demand Factors. Quarterly Journal of Economics, v.107, p. 35-72, 1992.

KURESKI, R.; NUÑEZ, B. E. C. Os multiplicadores de emprego e renda da indústria de papel e celulose 2003: uma aplicação da matriz de insumo-produto. Revista de Economia e Administração, v. 6, p. 8398, 2007.

MACHADO, A. F.; MOREIRA, M. M. Os Impactos da Abertura Comercial sobre a Remuneração do Trabalho no Brasil. In: ENCONTRO NACIONAL DE ECONOMIA, 28., 2000, Campinas. Anais...Campinas: ANPEC, 2000.

MACHIN, S. Changes in Relative Demand for Skills. In: BOOTH, A. L.; SNOWER, D. J. Acquiring Skills: Market Faulures, their Symptoms and Policy Responses. Cambridge: Cambridge University Press, 1996. 
MENEZES FILHO, N. A.; RODRIGUES JÚNIOR, M. Abertura, Tecnologia e Qualificação: Evidências para a Manufatura Brasileira. Workshop sobre Liberalização Comercial e Mercado de Trabalho no Brasil, Brasília, 2001.

MOREIRA, M. M.; NAJBERG, S. Abertura Comercial: Criando ou Exportando Empregos? Texto para Discussão n. 59, Banco Nacional de Desenvolvimento Econômico e Social. Rio de Janeiro, 1997.

NICKELL, S.; BELL, B. The Collapse in Demand for the Unskilled and Unemployment across the OECD. Oxford Review of Economic Policy. v.11, p. 40-62, 1995.

ROBBINS, J. D. Worsening Relative Wage Dispersion in Chile during Trade Liberalization, and its Causes: Is Supply at Fault? Development Discussion Paper n. 484, Harvard: Harvard Institute for International Development, 1994.

Stolper-Samuelson (Lost) in the Tropics? Trade Liberalization and Wages in Colombia: 1976 - 1994. Development Discussion Paper $n^{\circ} 563$ Harvard: Harvard Institute for International Development. 1996.

SACHS, J. D.; H. J. SHATZ, H. J. Trade and Jobs in U.S. Manufacturing. Brookings Papers on Economic Activity, v.1, p.1-84. 1994.

SARQUIS, S. J. B.; ARBACHE, J.S. Openess and External Effects of Humann Capital Mimeo. London School of Economics e Universidade de Brasília. 2001.

WOOD, A. North-South Trade, Employment and Inequality. Changing Fortunes in Skill-Driven World. Oxford: Clarendon Press. 1994. 